\title{
Pinch Analysis of Xylene Fractionation and CCR- Plateforming Process Using Aspen Plus
}

\author{
SUMIT GUJAR*, M.M.UPKARE and S.R.SATPUTE \\ Department of Chemical Engineering, Vishwarkarma Institute of Technology, Pune, India \\ sumitgujar001@gmail.com
}

Received 13 May 2015 / Accepted 4 June 2016

\begin{abstract}
This paper presents a case study of pinch analysis for xylene fractionation and CCRPlateforming processes which are highly energy intensive. A combination of 4 hot and 3 cold streams was considered for process integration. An Aspen Energy Analyser simulation was performed for the considered data. A minimum temperature difference $\left(\Delta \mathrm{T}_{\min }\right)$ was considered to be as $10^{\circ} \mathrm{C}$. Pinch temperature was found to be $103{ }^{\circ} \mathrm{C}$ and hot utility usage was found to be reduced by $53.01 \mathrm{~kW}$. The initial investment was found to be Rs $2.49 * 10^{\wedge} 6$ while profit generated was found to be $2.79 * 10^{\wedge} 6$ giving a payback period of 10 months.
\end{abstract}

Keywords: Pinch analysis, Process intensification, Aspen energy analyser, Energy targeting

\section{Introduction}

Production of aromatics has been used to obtain large number of commercially useful sub products. However aromatics production is extremely energy intensive. The aromatics plant studied consist of different units with huge energy requirement. Process integration attempts to reduce these energy requirements. A useful tool for the process integration is pinch technique. Over the time, this technology has proven to be the most reliable and efficient ${ }^{1}$.

Pinch analysis is a methodology for minimising energy consumption of chemical processes by calculating thermodynamically feasible energy targets (minimum energy consumption and achieving them by optimising the heat recovery systems, energy supply methods and process operating conditions. This technology is useful when integrating heat exchanger networks in chemical plants as it reduces capital costs and decreases specific energy demands.

In this work we have made an attempt to study the application of pinch analysis for the particular process intensification problem. 


\section{Experimental}

\section{Pinch technique}

Pinch technique was developed by Bodo Linhoff in late 1980's ${ }^{2}$. This technique was based on thermodynamic principles and allows to determine the best heat exchanger and utility systems. The key step in this technique is to consider a proper heat integration data. This is known as data extraction. Results derived from this technique mainly depend on the data. Data extraction involves different combination of hot and cold streams which can be integrated to reduce utility usage. Here attempt is made to maximise process-process heat exchange and minimize process-utility loads.

As each stream can be represented on $\mathrm{T}-\mathrm{H}$ curve, hot streams and cold streams were overlapped on T-H diagram to obtain pinch point. The overshoot of these curves give thermodynamically minimum possible utility requirement and provide energy targets for the process based on only the stream data as it is depicted in Figure 1.

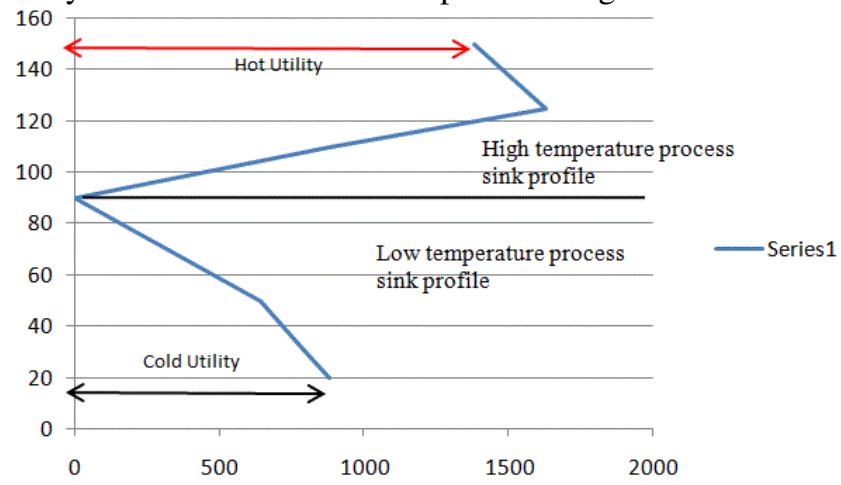

Figure 1. Composite curve showing Pinch Technique ${ }^{3}$

\section{Results and Discussion}

\section{Data extraction}

In the this work combination of 2 different units having 4 hot and 3 cold streams in total was considered. Additionally two utilities from aspen energy analyser data base have been included to satisfy the energy requirement as outlined in Table 1.

Table 1. Problem statement with 4 hot and 3 cold streams

\begin{tabular}{cccccc}
\hline Stream & Condition & Tin, ${ }^{\circ} \mathrm{C}$ & Tout, ${ }^{\circ} \mathrm{C}$ & $\mathrm{M}^{*} \mathrm{C}_{\mathrm{p}}, \mathrm{kW} /{ }^{\circ} \mathrm{C}$ & Enthalpy, $\mathrm{kW}$ \\
\hline 1 & Hot & 67 & 38 & 28.87 & 837.2 \\
2 & Cold & 176 & 180 & 2802 & 11208.64 \\
3 & Hot & 74 & 38 & 4.884 & 174.4 \\
4 & Hot & 108 & 38 & 2.325 & 162.8 \\
5 & Hot & 53 & 40 & 5.769 & 75 \\
6 & Cold & 34 & 149 & 0.7088 & 81.51 \\
7 & Cold & 53 & 139 & 0.3109 & 26.74 \\
\hline
\end{tabular}

Basic utilities were HP steam and cooling water. HP steam available at $250{ }^{\circ} \mathrm{C}$ and leaving as $249{ }^{\circ} \mathrm{C}$ where as cooling water was at $20{ }^{\circ} \mathrm{C}$ and leaving at $25^{\circ} \mathrm{C}$. In order to avoid high investment costs, in practice a minimum temperature difference $\left(\Delta \mathrm{T}_{\min }\right)$ at the pinch point was demanded, e.g., $10{ }^{\circ} \mathrm{C}$. 


\section{Energy targeting}

Energy targeting was obtained by shifted temperatures using following equations.

$$
\begin{gathered}
\text { For Hot Streams: } T_{\mathrm{int}}=T_{a c t}-\Delta T_{\min } / 2 \\
\text { For Cold Streams: } T_{\mathrm{int}}=T_{a c t}+\Delta T_{\min } / 2
\end{gathered}
$$

Shifted temperatures were arranged in descending order omitting the common values. When temperatures were arranged in descending order, temperature interval diagram was plotted to obtain location of different streams in each interval as described in Figure 2. Resultant CP was calculated from this diagram. For each interval, heat load was calculated. This provided cascaded heat and revised cascaded heat load was calculated using below formulas and are described in Figure 3.

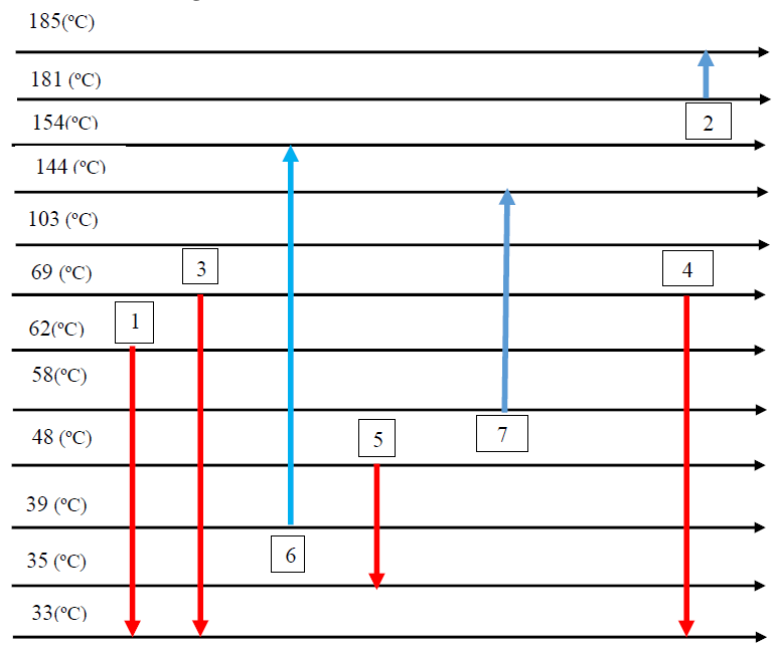

\begin{tabular}{|c|c|c|c|c|c|c|}
\hline \multicolumn{3}{|c|}{ Infeasible Cascade } & \multicolumn{2}{|c|}{ Feasible Cascade } & \multicolumn{2}{|c|}{ Shift Temperature } \\
\hline & \multirow[b]{2}{*}{$\boldsymbol{\nabla}$} & Qcas $(\mathrm{kW})$ & & \multicolumn{2}{|c|}{ Rcas $(\mathrm{kW})$} & \\
\hline & & 0 & $\boldsymbol{\nabla}$ & 11256.9 & 185 & \\
\hline & 11208 & & 11208 & & & \\
\hline & $\nabla$ & -11208 & $\nabla$ & 48.8957 & 181 & \\
\hline & 0 & & 0 & & & \\
\hline & $\nabla$ & -11208 & $\nabla$ & 48.8957 & 154 & \\
\hline & 7.088 & & 7.088 & & & \\
\hline & $\boldsymbol{\nabla}$ & -11215 & $\boldsymbol{\nabla}$ & 41.806 & 144 & \\
\hline & 41.8077 & & 41.8077 & & & \\
\hline $\mathrm{PINCH}$ & $\boldsymbol{\nabla}$ & -11257 & $\boldsymbol{\nabla}$ & 0 & 103 & \\
\hline & -44.3802 & & -44.3802 & & & \\
\hline & $\nabla$ & -11213 & $\nabla$ & 44.38 & 69 & \\
\hline & -43.3251 & & -43.3251 & & & \\
\hline & $\boldsymbol{\nabla}$ & -11169 & $\boldsymbol{\nabla}$ & 87.71 & 62 & \\
\hline & -140.237 & & -140.237 & & & \\
\hline & $\nabla$ & -11029 & $\nabla$ & 227.95 & 58 & \\
\hline & -347.484 & & -347.484 & & & \\
\hline & $\nabla$ & -10681 & $\nabla$ & 575.43 & 48 & \\
\hline & -364.657 & & -364.657 & & & \\
\hline & $\boldsymbol{\nabla}$ & -10317 & $\nabla$ & 940.09 & 39 & \\
\hline & -159.234 & & -159.234 & & & \\
\hline & $\nabla$ & -10158 & $\nabla$ & 1099.32 & 35 & \\
\hline & -68.0792 & & -68.0792 & & & \\
\hline & $\nabla$ & -10090 & $\nabla$ & 1167.4 & 33 & \\
\hline
\end{tabular}

Figure 2. Interval Temperature Diagram

Figure 3. Cascade Diagram 


$$
\begin{aligned}
Q_{\mathrm{int}} & =\Delta T_{\mathrm{int}} *\left(\Sigma C_{p, c}-\Sigma C_{p, h}\right)_{\mathrm{m}} \\
Q_{\text {cas }, i} & =Q_{\text {cas }, i-1}-Q_{\mathrm{int}, i} \\
R_{\text {cas }, i} & =Q_{\text {cas }, i}-\min \left(Q_{\text {cas }}\right)
\end{aligned}
$$

For $e . g .:$ for $1^{\text {st }}$ interval: $\mathrm{C}_{\mathrm{p}}$ of $4^{\text {th }}$ stream* Temperature Difference $=-311.4074 * 46=-$ $14325 \mathrm{~kW}$. Same procedure was repeated for all the balance intervals and the $\mathrm{Q}_{\text {int }}$ values were obtained. Results are summarised in Table 2 . Nil value in $R_{\text {cas }}$ column indicates 'Pinch Point'. The first value indicates minimum hot utility and the last value indicates minimum cold utility requirement.

Table 2. Detailed Calculations

\begin{tabular}{cccccc}
\hline Interval & Temperature, ${ }^{\circ} \mathrm{C}$ & $\mathrm{MC}_{\mathrm{p}}\left(\mathrm{kW} /{ }^{\circ} \mathrm{C}\right)$ & $\mathrm{Q}_{\text {int }}(\mathrm{kW})$ & $\mathrm{Q}_{\text {cas }}(\mathrm{kW})$ & $\mathrm{R}_{\text {cas }}(\mathrm{kW})$ \\
\hline 0 & 185 & 0 & 0 & 0 & 11256.9 \\
1 & 181 & 2802 & 11208 & -11208 & 48.8957 \\
2 & 154 & 0 & 0 & -11208 & 48.8957 \\
3 & 144 & 0.7088 & 7.088 & -11215.09 & 41.806 \\
4 & 103 & 1.0197 & 41.8077 & -11256.9 & 0 \\
5 & 69 & 1.0197 & -44.3802 & -11212.52 & 44.38 \\
6 & 62 & -6.1893 & -43.3251 & -11169.19 & 87.11 \\
7 & 58 & -35.0593 & -140.237 & -11208.95 & 227.95 \\
8 & 48 & -34.7484 & -347.484 & -10681.47 & 575.43 \\
9 & 39 & -40.5174 & -364.657 & -10316.81 & 940.09 \\
10 & 35 & -39.8086 & -159.234 & -10157.58 & 1099.32 \\
11 & 33 & -34.0396 & -68.0792 & -68.0792 & 1167.396 \\
\hline
\end{tabular}

Summary of energy targeting results:, Pinch temperature $=103{ }^{\circ} \mathrm{C}$, Hot utility requirement $=11256.9$ $k W$, Cold utility requirement $=1167.396 \mathrm{~kW}$

\section{Heat exchanger specifications}

The heat exchanger network was generated by using aspen energy analyser. Figure 4 gives detail about the structure.

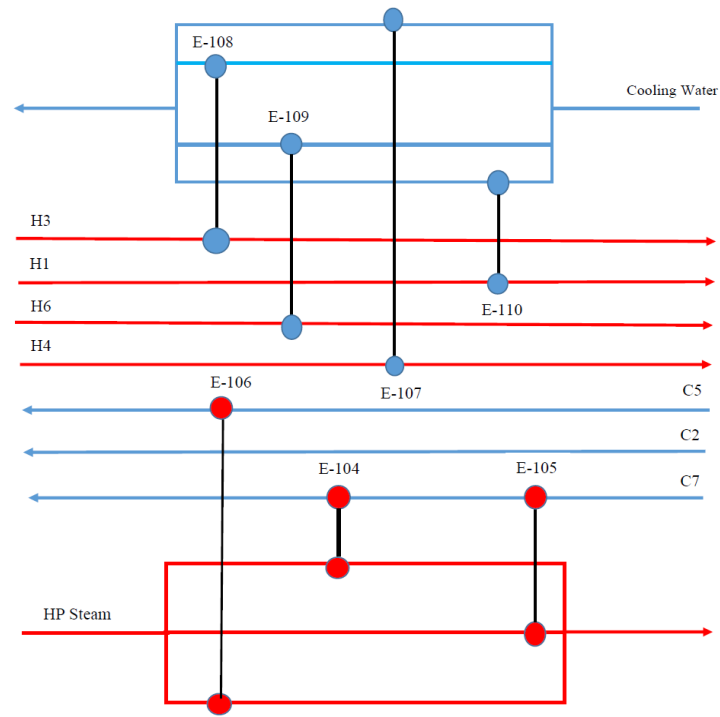

Figure 4. Proposed heat exchanger network 
Details for all the heat exchangers are described in Table 3 and Table 4 respectively.

Table 3. Stream data for all heat exchangers

\begin{tabular}{|c|c|c|c|c|c|c|c|c|c|c|c|}
\hline & Cooler/ & Cold & Cold Tin, & & Cold & & Hot & Hot Tin, & & Iot Tor & \\
\hline Exchanger & Heater & Stream & ${ }^{\circ} \mathrm{C}$ & Tied & Tout, ${ }^{\circ} \mathrm{C}$ & Tied & Stream & ${ }^{\circ} \mathrm{C}$ & Tied & ${ }^{\circ} \mathrm{C}$ & Tied \\
\hline E-110 & Cooler & $\begin{array}{c}\text { Cooling } \\
\text { water }\end{array}$ & 20 & & 25 & & $\mathrm{H} 1$ & 67 & $\mathrm{~T}$ & 38 & $\mathrm{~T}$ \\
\hline E-105 & Heater & $\mathrm{C} 2$ & 176 & $\mathrm{~T}$ & 180 & $\mathrm{~T}$ & $\begin{array}{c}\text { HP } \\
\text { Steam }\end{array}$ & 250 & & 249 & \\
\hline E-108 & Cooler & $\begin{array}{c}\text { Cooling } \\
\text { water }\end{array}$ & 20 & & 25 & $\mathrm{~T}$ & $\mathrm{H} 3$ & 74 & $\mathrm{~T}$ & 38 & $\mathrm{~T}$ \\
\hline E-106 & Heater & C6 & 34 & $\mathrm{~T}$ & 149 & & $\begin{array}{c}\text { HP } \\
\text { Steam }\end{array}$ & 250 & & 249 & \\
\hline E-109 & Cooler & $\begin{array}{c}\text { Cooling } \\
\text { water }\end{array}$ & 20 & & 25 & & $\mathrm{H} 5$ & 53 & $\mathrm{~T}$ & 40 & $\mathrm{~T}$ \\
\hline E-107 & Cooler & $\begin{array}{c}\text { Cooling } \\
\text { water }\end{array}$ & 20 & & 25 & & $\mathrm{H} 4$ & 108 & $\mathrm{~T}$ & 38 & $\mathrm{~T}$ \\
\hline E-104 & Heater & C7 & 53 & $\mathrm{~T}$ & 139 & $\mathrm{~T}$ & $\begin{array}{c}\text { HP } \\
\text { Steam }\end{array}$ & 250 & & 249 & \\
\hline
\end{tabular}

Table 4. Key parameters for all the heat exchangers

\begin{tabular}{cccccccc}
\hline $\begin{array}{c}\text { Heat } \\
\text { Exchanger }\end{array}$ & $\begin{array}{c}\text { Load } \\
(\mathrm{kW})\end{array}$ & Cost Index & Area $\left(\mathrm{m}^{2}\right)$ & Shells & $\begin{array}{c}\text { LMTD } \\
\left({ }^{\circ} \mathrm{C}\right)\end{array}$ & $\begin{array}{c}\text { Overall U*10 } \\
\left(\mathrm{kW} / \mathrm{m}^{2}{ }^{\circ} \mathrm{C}\right)\end{array}$ & $\begin{array}{c}\mathrm{F} \\
\text { factor }\end{array}$ \\
\hline E-110 & 837.16 & 56574.6276 & 160.814148 & 1 & 28.32 & 18.98 & 0.96 \\
E-105 & 11208.64 & 205055.4805 & 810.172904 & 2 & 71.48 & 19.35 & 0.99 \\
E-108 & 174.4 & 22386.30543 & 30.7124702 & 2 & 30.95 & 18.98 & 0.96 \\
E-106 & 81.51 & 11819.70125 & 2.79342896 & 1 & 150.88 & 19.35 & 0.99 \\
E-109 & 75 & 17697.07164 & 16.9451114 & 1 & 23.77 & 18.98 & 0.98 \\
E-107 & 162.8 & 19119.84216 & 20.9469921 & 1 & 42.52 & 18.98 & 0.96 \\
E-104 & 26.74 & 10751.47574 & 0.92476465 & 1 & 149.49 & 19.35 & 0.99 \\
\hline
\end{tabular}

In this way, all the important parameters for heat exchangers were developed to generate the heat exchanger network (HEN).

\section{Cost estimation}

Unless and until any modifications are yielding profits, there should not be any modifications in current systems. Modified systems should be economically feasible. In the work initially amount of hot and cold utility required were $11316.59 \mathrm{~kW}$ and $1249 \mathrm{~kW}$ respectively.

After performing pinch analysis, there was considerable reduction in utility requirements. Superstructure for the given system was generated showing hot utility usage of $11263.88 \mathrm{~kW}$ and cold utility usage of $1197 \mathrm{~kW}$. Superstructure can be implemented practically as Aspen takes almost all practical situations into considerations. Profit can be calculated from the difference between hot utility requirements initially and after superstructure. Currently utility is charged at $1 \mathrm{KWh} / \mathrm{Day}$ is Rs 6 and plant is considered to be operated $24 * 7 * 365$ days. 
Hence, Profit $=(11316.89-11263.88) * 6 * 24 * 365$

$$
=\text { Rs. } 2.79 * 10^{6} / \text { year }
$$

To effect the revised structure, following over head were considered. Total piping required $250 \mathrm{~m}$. A 106 grade B pipe (5 in) was proposed. Thus pipe weight per meter is $21.77 \mathrm{~kg} / \mathrm{m}$.

The pipe cost was approximated at $300 \mathrm{Rs} / \mathrm{kg}$, Pipe require $=250 \mathrm{mx} 21.77 \mathrm{~kg} / \mathrm{m}=$ $5442.5 \mathrm{~kg}=5.44 \mathrm{ton}$, Required cost for piping $=5442.5 \times 300=\mathrm{Rs} 1.63 * 10^{6}$, we take over head cost $50 \%$ of that

Total piping cost required $=1.63 * 10^{6}+1.63 * 10^{6} \times 0.50$

Payback period

$$
=\text { Rs. } 2.49 * 10^{6}
$$

Payback period is the time in which the initial cash outflow of an investment is expected to be recovered from the cash inflows generated by the investment.

Payback period $=$ initial investment $/$ cash inflow per year

$$
\begin{aligned}
& =2.49 * 10^{6} / 2.79 * 10^{6} \text { year } \\
& =0.87 \text { year }(10 \text { months })
\end{aligned}
$$

Total piping cost required was Rs 24.5 lakh and our annual net saving was Rs 27 lakh hence the payback period will be 0.87 year or 10 months. After 0.87 year or 10 months profit will start.

Energy savings remains to be a vital part in process modifications. There is considerable drop in amount of utility usage and some key worthy modifications. The amount of hot utility required is $11263 \mathrm{~kW}$ and pinch point is obtained at $103{ }^{\circ} \mathrm{C}$. Area required for counter-current is $1056 \mathrm{~m}^{2}$ and for 1-2 Shell and Tube heat exchangers it is $1062 \mathrm{~m}^{2}$. Minimum number of units required for MER are 9 and shells required are 9. Initial investment is of Rs. $2.49 * 10^{6}$ whereas cash inflow is Rs. $2.79 * 10^{6}$ giving payback period of 0.87 year or 10 months after which profit can be generated.

\section{Conclusion}

The heat integration with xylene fractionation and CCR- Plate forming process unit model is studied by ASPENHX-NET simulator with the constraints of the maximum energy recovery and minimum heat transfer area. Heat exchanger network was effectively designed by pinch table algorithm. A considerable drop was energy requirement was found giving profit of Rs. $2.79 * 10^{6}$. The cost required for retrofitting is Rs $2.49^{*} 10^{6}$. The method was successfully used to allow trade-offs in energy between a number of heat medium units with little net capital outlay.

\section{References}

1. Morgan S, Use of Process Integration to Improve Process Designs and Design Process, Chemical Engineering Progress, September, 1992, pp.62-68.

2. Linnhoff T B and Hindmarsh E, Chem Engg Sci., 1983, 38(5), 745-763; DOI:10.1016/0009-2509(83)80185-7

3. Kerner H, Chemie Ingenieur Technik, 1988, 60(7), 511-518; DOI:10.1002/cite.330600703

4. Shenoy UV, Heat Exchanger Networks Synthesis, USA, 1995, pp.2-48.

5. Kemp I C, Pinch Analysis and Process Integration, $2^{\text {nd }}$ Edition, pp.15-27.

6. McCabe W L, Smith J C, Unit Operations of Chemical Engineering $5^{\text {th }}$ Edition, 1993 pp.427-439.

7. Sinnott R K, Coulson and Richardson's, Chemical Engineering, Chemical Engineering Design, Volume 6, $3^{\text {rd }}$ edition., Butterworth-Heinemann, Great Britain, 1999, pp.102-128. 